\title{
Wallerian Degeneration in the Corticospinal Tract Following Tumefactive Demyelination: Conventional and Advanced Magnetic Resonance Imaging
}

\author{
Todd A. Hardy, Chenyu Wang, Heidi N. Beadnall, Jim Lagopoulos, Yael Barnett, \\ Alexander Klistorner, Michael H. Barnett
}

Keywords: Multiple sclerosis, pseudotumor, tumor-like, MRI, tractography, axonal degeneration, tumefactive demyelination

A 24-year-old man with no prior medical history or neurological symptoms presented with the subacute onset of dysarthria and mild right-sided weakness involving the face, arm, and leg. On examination, the only abnormalities were a right upper motor neurone facial droop and mild right pyramidal weakness. 3-Tesla magnetic resonance imaging (MRI) of the brain revealed a large, isolated, nonenhancing, peripherally diffusion-restricted T2 hyperintense lesion in the left frontal lobe with minimal edema and mass effect; and a T2 hypointense rim, typical of a tumefactive demyelinating lesion (TDL) (Figure 1A,B). MRI of the whole spine was normal. Oligoclonal bands in the cerebrospinal fluid were positive and visual evoked potentials were mildly prolonged bilaterally, consistent with demyelination. Serum aquaporin -4 antibody was negative. He was treated with intravenous methylprednisolone $1 \mathrm{~g}$ daily for 5 days, followed by interferon beta $-1 \mathrm{a}$. His neurological deficits improved to normal over several months.

Repeat imaging 2 years later showed involution of the TDL, with MRI changes in the left corticospinal tract (CST) consistent with Wallerian degeneration (WD) (Figure 1C). Application of diffusion tensor imaging (DTI) using probabilistic tractography (Figure 1D,E) showed higher axial diffusivity (AD; 1.26 vs $1.07 \mu \mathrm{m}^{2} / \mathrm{ms}$ ) and radial diffusivity (RD; $0.75 \mathrm{vs} 0.59 \mu \mathrm{m}^{2} / \mathrm{ms}$ ) in the affected CST compared with the normal-appearing contralateral side. Moderate increases of AD $\left(1.23 \mathrm{vs} 1.16 \mu \mathrm{m}^{2} / \mathrm{ms}\right)$ and RD (0.58 vs $\left.0.52 \mu \mathrm{m}^{2} / \mathrm{ms}\right)$ were observed in the tract proximal to the lesion, whereas higher $\mathrm{AD}\left(1.40 \mathrm{vs} 1.34 \mu \mathrm{m}^{2} / \mathrm{ms}\right)$ but similar RD ( $\left.0.45 \mathrm{vs} 0.46 \mu \mathrm{m}^{2} / \mathrm{ms}\right)$ was measured in the CST distal to the lesion. Four years later, the patient has no neurological symptoms and remains clinically and radiologically stable.

\section{Discussion}

TDLs are an uncommon manifestation of demyelinating disease, often mistaken for neoplasm. ${ }^{1,2}$ MRI clues that help to distinguish TDLs include size $>2 \mathrm{~cm}$, open ring enhancement, diffusion restriction at the lesion periphery, a T2 hypointense rim around a T2 hyperintense core, venular enhancement, and comparatively minimal paralesional edema and mass effect for size. $^{2,3}$ Not all lesions will necessarily have all these features, and close clinical and radiological follow-up is advised. In some cases, a brain biopsy may be required to exclude alternative pathologies. 1,2

Acute demyelinating lesions can cause transection of axons leading to distal axonal WD. ${ }^{4}$ Although we might have expected to see a residual right hemiparesis, or even progressive weakness from this lesion as a result of the WD, the patient has a normal and stable neurological examination. Presumably this reflects that a critical number of fibers within the CST have remained functional above a threshold for clinical weakness and/or that power has been maintained by the recruitment of additional neural pathways.

This is the first description of WD explicitly resulting from tumefactive demyelination, although some TDLs were included in an earlier conventional MRI study that also identified WD in the CST of patients with demyelinating lesions of different sizes. ${ }^{5}$ MRI poorly resolves anterograde axonal loss from small individual demyelinating lesions, but the size of TDLs involving the CST makes it possible to visualize the WD that occurs after transection of a larger number of axons. ${ }^{5}$

Debate exists as to whether changes in diffusivity are related to axonal loss, demyelination, or some combination of the two. ${ }^{6}$ DTI studies are limited by a lack of knowledge regarding the precise pathology of the area being studied in vivo and that there are often coexistent and overlapping areas of demyelination and axonal degeneration. Moreover, other fiber tracts can cross across through an area of interest, and $\mathrm{AD}$ and $\mathrm{RD}$ values can change over time in relation to an acute demyelinating event. ${ }^{6}$ Changes in diffusivity therefore are likely to be more robust across chronically affected larger fiber tracts such as the CST, where these factors should have less impact. Ongoing studies with radio-pathological correlation and improvement in DTI metrics will help to resolve the debate.

From the Brain \& Mind Centre, University of Sydney, NSW, Australia

(TAH, CW, HNB, JL, AK, MHB); Neuroimmunology Clinic, Concord Repatriation General Hospital, University of Sydney, NSW, Australia (TAH); Sydney Neuroimaging Analysis Centre, Sydney, NSW, Australia (CW, YB, AK, MHB); Department of Radiology, St Vincent's Hospital, University of NSW, Australia (YB); Department of Neurology, Royal Prince Alfred Hospital, Sydney, NSW, Australia (MHB).

Received July 18, 2015. Final Revisions Submitted January 7, 2016. Date of AcCePtAnCE March 28, 2016.

Correspondence to: Todd A. Hardy, Brain \& Mind Centre, University of Sydney, NSW, 2050, Australia. Email: thardy@med.usyd.edu.au 

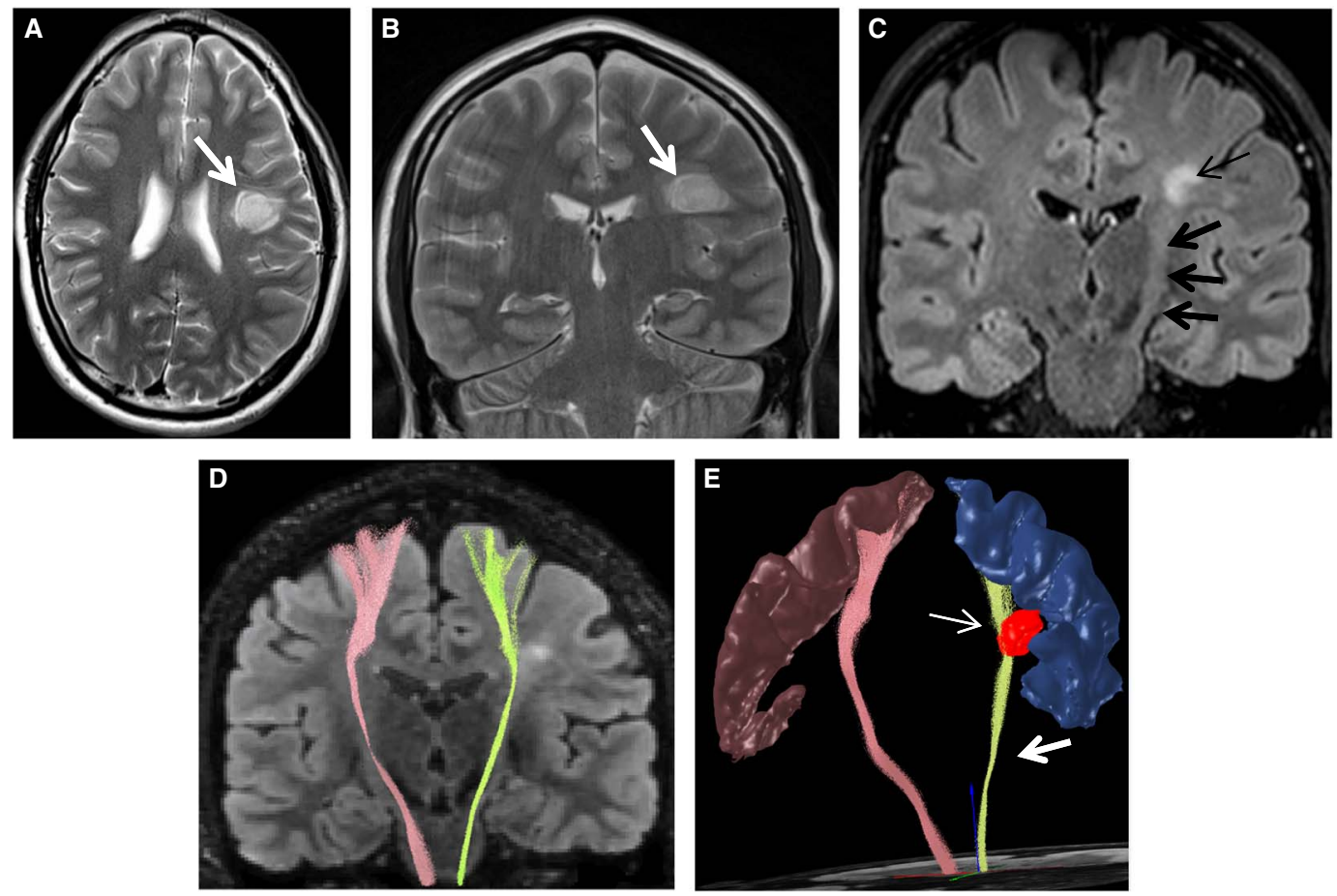

Figure 1: Magnetic resonance imaging (A) axial T2 and (B) coronal T2 showing a tumefactive demyelinating lesion (TDL) in the left frontal lobe (arrows). (C) Coronal fluid attenuated inversion recovery image 2 years later shows involution of the lesion (thin arrow) with distal signal change in the path of the corticospinal tract (CST) consistent with Wallerian degeneration (thick arrows). (D) Probabilistic tractography (PT)-based fiber reconstruction comparing the normal appearing CST on the right (pink) with the lesional side on the left (green). (E) PT-delineated CST shows the presumed area of Wallerian degeneration (short thick arrow) below the lesion (thin arrow; red) on the left. The PT-delineated CSTs are shown in relation to associated areas of cortex on the right (brown) and left (blue). Images are radiologically oriented and color-coded.

Chronic axonal degeneration resulting from acute demyelinating lesions is thought to be a major factor underlying multiple sclerosis disease progression. ${ }^{3}$ In this patient, further chronic axonal degeneration, or the development of new focal lesions, may exhaust axonal reserve in the CST and result in early secondary progressive weakness.

\section{Disclosures}

The authors do not have anything to disclose.

\section{REFERENCES}

1. Hardy TA, Chataway J. Tumefactive demyelination: an approach to diagnosis and management. J Neurol Neurosurg Psychiatry. 2013;84:1047-53.
2. Lucchinetti CF, Gavrilova RH, Metz I, et al. Clinical and radiographic spectrum of pathologically confirmed tumefactive multiple sclerosis. Brain. 2008;131:1759-75.

3. Dutta R, Trapp BD. Pathogenesis of axonal and neuronal damage in multiple sclerosis. Neurology. 2007;68:S22-31; discussion S43-54.

4. Kiriyama T, Kataoka H, Taoka T, et al. Characteristic neuroimaging in patients with tumefactive demyelinating lesions exceeding $30 \mathrm{~mm}$. J Neuroimaging. 2011;21:e69-77.

5. Simon JH, Kinkel RP, Jacobs L, et al. A Wallerian degeneration pattern in patients at risk for MS. Neurology. 2000;54: 1155-60.

6. Rocca MA, Mesaros S, Preziosa P, et al. Wallerian and trans-synaptic degeneration contribute to optic radiation damage in multiple sclerosis: a diffusion tensor MRI study. Mult Scler. 2013;19: 1610-7. 